\title{
Methods of Fractal Analysis Applied to STM Imaging
}

\author{
L. VAZQUEZ," J.M. VARA," P. HERRASTI," P. OCON," R.C. SALVAREZZA \\ and
}

\section{A.J. ARVIA}

INIFTA, Instituto de Investigaciones Fisicoquímicas Teóricas y Aplicadas, UNLP-CONICET, Sucursal 4, Casilla de Correo 16, (1900) La Plata, Argentina.

*) Visiting Professor from Instituto de Ciencia de Materiales, Universidad Autónoma de Madrid C-XII, 28049 Madrid, Spain.

\#)Dpto. Química Física Aplicada. Universidad Autónoma de Madrid, C-II. 28049 Madrid, Spain.

\begin{abstract}
Vapor deposited gold films have been characterized by applying methods of fractal analysis to scanning tunneling microscopy (STM) images. Results from the use of five different methods are in agreement within the limitation of each method. Those methods are suitable to characterize rough surfaces at the nanometer level provided that a large number of images is considered.
\end{abstract}

\section{INTRODUCTION}

The relationship between surface growth and fractal properties has been an important subject of research in recent years [1], particularly much effort has been devoted to develop computer simulation models of surface growth. These efforts contrast with the lack of sufficient experimental data on topographies at the $\mathrm{nm}$ level which are necessary to check the predictions of models. The recent application of scanning tunneling microscopy (STM) [2-6] to investigate topographies of solid objects offers the possibility of supplying the corresponding data. Several methods based on the analysis of STM images have been proposed for a quantitative description of irregular surfaces. However, results from different methods applied to a common surface present some discrepancies [7]. In this paper we report on the application of five different methods for fractal analysis to STM images of gold films. Data allowed us to set the limitation and validity ranges of each method. The dynamic scaling of surface growth can be applied to characterize rough surfaces [1]. In this case, for a sample of lateral dimension $L$ formed by $N$ points, $\xi(L)$, the surface width in the i-direction, which defines the root mean square roughness, is given by

$$
\xi(L)=\left[1 / N \sum\left(h_{j}-H\right)^{2}\right]^{1 / 2}
$$

where $h_{j}$ is the deposit height measured along the i-direction at the j-position, and $\mathrm{H}$ is the average height of the sample. It has been shown that $\xi(\mathrm{L})$ in Eq. (1) reaches a steady value after a certain critical thickness (growth time). Then, $\xi(\mathrm{L})$ and $\mathrm{L}$ fit the proportionality

$$
\xi(\mathrm{L}) \propto \mathrm{L}^{\alpha}
$$

where exponent $\alpha$ is a relevant quantity related to the local fractal dimension of the self affine surface [1]. 
Single Image Dynamic Scaling STM methods (SIDS). The dynamic scaling theory [1] can be directly applied to STM data by setting $\xi\left(L_{S}\right)=\xi_{\text {stm }}\left(L_{S}\right)$, where $\xi_{\text {stm }}\left(L_{S}\right)$ is the root mean square roughness determined by STM scans in the $x$-direction. Then, the following equations can be used [5]:

$$
\xi_{s t m}\left(L_{s}\right) \propto\left[\sum\left(h_{j}-h_{s}\right)^{2}\right]^{1 / 2}
$$

and

$$
\xi_{\text {stm }}\left(\mathrm{L}_{\mathrm{s}}\right) \propto \mathrm{L}_{\mathrm{s}}^{\alpha}
$$

where $L_{S}$ is the length measured along $S$, the STM scan length, and $h_{S}$ is the average height of the surface profile of length $L_{S}$ determined by STM scans in the $x$-direction.

Multiple Image Dynamic-Scaling-STM (MIDS). A variation of SIDS is the multiple image dynamic-scaling -STM method which involves the use of both the value of $\xi_{\text {stm }}$ obtained from each STM image, and the value of $\mathrm{S}$ as variables $[4,6]$. These values fit the proportionality:

$$
\xi_{\text {stm }}(S)=S^{\alpha}
$$

The Variogram method (V). The properties of saturated interfaces can be obtained by using $c(L)$, the heightto-height correlation function, defined by:

$$
c\left(L_{s}\right)=<\left|h\left(L_{s}+x\right)-h(x)\right|^{2}>
$$

Otherwise, $c\left(L_{S}\right)$ is related to $\alpha$ through the proportionality

$$
\mathrm{c}\left(\mathrm{L}_{\mathrm{s}}\right) \propto \mathrm{L}_{\mathrm{s}}^{2 \alpha}
$$

The Fourier Transform method (FT). The FT method is based on Fourier transform analysis [2]. P(K), the scaling power spectra, of a sel-affine fractal topographic profile, has the form

$$
\mathrm{P}(\mathrm{K}) \propto \mathrm{K}^{-(2 \alpha-1)}
$$

where $K$ is the wavenumber. Then, the value of $\alpha$ can be derived from the slope of the $\log (P)$ vs $\log K$ plot. To improve the method performance [2] every STM profile is subjected to a Fourier transform routine, all the transforms are averaged, and IP, the power spectra integrated from high to low frequencies is obtained. From the slope of the $\log (\mathrm{IP})$ vs $\log (\mathrm{K})$ plot, the $\alpha$ value can be derived.

The Perimeter-Area method (PA). The PA method [3] considers that the intersection of a plane with a selfaffine or a self-similar fractal surface generates self-similar lakes or islands [8] for which the following proportionality between $P$, the perimeter, and $A$, the area of these lakes or islands, is observed

$$
\mathrm{P} \propto \mathrm{A}^{D / 2}
$$

where

$$
\alpha=2-D_{L}^{\prime}
$$

$D^{\prime} L$ is the fractal dimension of either the island or lake perimeter. It should be noted that for measuring a rough profile the yardstick must be smaller than the size of the smallest irregularity [4].

The capability of the above mentioned methods was evaluated through their application to the analysis of a number of computer generated surface profiles which were obtained using a random addition algorithm [9] with known values of $\alpha$. For typical STM file sizes, the relationship between $\alpha$, the experimental value, and $\alpha_{\mathrm{th}}$, the value expected from the different methods, is shown in Fig. 1 [7]. Thus, for $\alpha>0.7$ all methods underestimate the value of $\alpha$, and for $\alpha<0.7$ it seems that the variogram method [7] yields the best results, whereas the FT appears to be the poorest method [2,7]. It is known that the difference $\alpha-\alpha_{\text {th }}$ can be diminished as the number of data points is increased [3]. However, this situation implies longer acquisition times in which the drift of the STM itself can produce image distortion, turning this possibility uncertain with 
the present STM facilities. Hence, the plot shown in Fig. 1 is used to derive the true $\alpha$ value.

Otherwise, STM images usually involve a plane (or slope) superimposed to the true morphological surface profile coming from the tip displacement from the normal to the sample surface. This plane has to be removed for fractal surface analysis [6], by employing the standard STM software. STM images scanned along the $\mathrm{x}$-direction, i.e. the fastest scanning direction, are preferentially used for the application of fractal analysis. STM images alone the $y$-direction, i.e. the slowest scanning direction, can provide wrong results due to drift distortions and noise problems [3,5]. In some cases, the STM imaging can also be influenced by the tip morphology itself, although this problem can be overcome by using different tips to image the same sample.

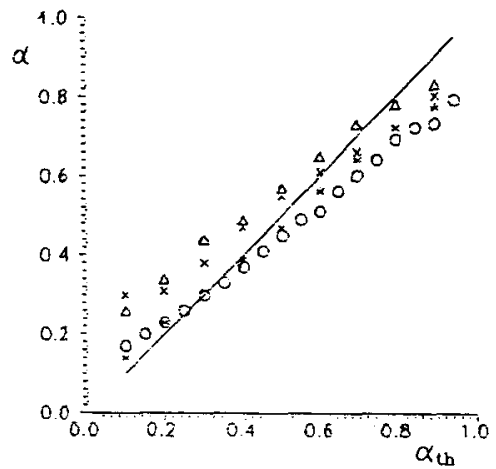

Fig.1. $\alpha$ vs $\alpha_{\text {th }}$ plots from SIDS (0), V (*), PA ( $\left.\Delta\right)$ and FT $(\mathrm{X})$ methods.

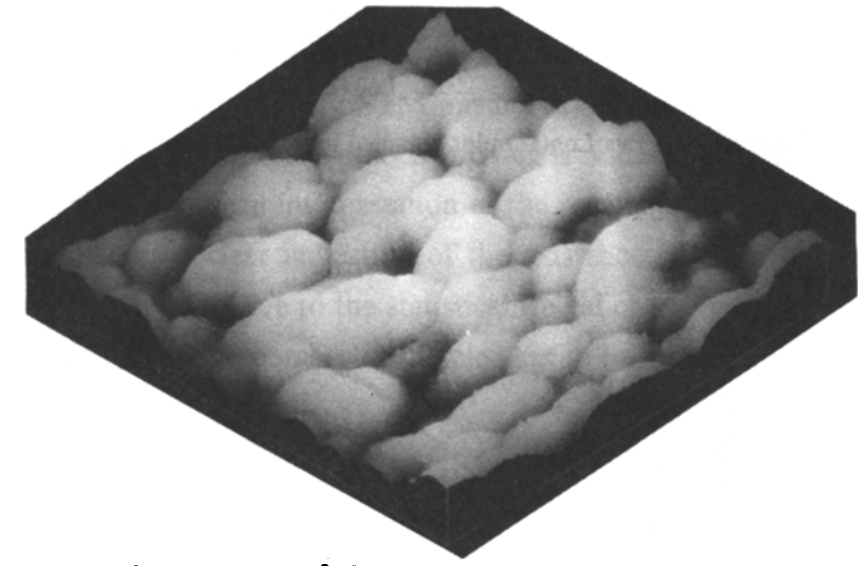

Fig. $2.600 \times 600 \mathrm{~nm}^{2}$ STM image of a $850 \mathrm{~nm}$ thick gold film.

\section{EXPERIMENTAL}

STM images of vapor deposited gold films ( $850 \mathrm{~nm}$ in thickness) grown at a high growth rate (30 nm $\mathrm{s}^{-1}$ ) on glass, were taken with a Nanoscope II and a McAllister equipment operating in air. From previous works [4,5] it is known that gold films of that thickness have already reached the $\xi(\mathrm{L})$ steady value required for the evaluation of $\alpha$. STM images consist of 400 scanlines composed of 400 data points. Vapor deposited gold films show a large number of small rounded elements which correspond to column tips that agglomerate to form large columns (Fig. 2). At a higher resolution, the surface of the smallest size column tips appear smooth with $d_{s}$, the average columnar size, close to $20-30 \mathrm{~nm}$.

\section{RESULTS}

Results obtained from the application of fractal analysis methods to STM images of vapor deposited gold films on glass are shown in Figs.3a-e. With the exception of the MIDS method, results from other methods show two linear regions ( $I$ and II) in the corresponding $\log -\log$ plot with a crossing point at $L_{S C} \approx d_{S}$. The values of $\alpha(\mathrm{I})$ and $\alpha(\mathrm{II})$ are obtained from the slopes of the straight lines. The final $\alpha$ values after correction (Fig.1) and the $\mathrm{L}_{\mathbf{S c}}$ values (averaged for 50-100 images) are shown in Table $\mathrm{I}$. The final $\alpha$ values resulting from both regions (Table I) are $\alpha(\mathrm{I})=0.75 \pm 0.1$ and $\alpha(\mathrm{II})=0.33 \pm 0.05$ for all methods. The main deviation for $\alpha(\mathrm{I})$ comes out from the SIDS method. The absence of region (I) in the log-log plot resulting from MIDS is due to the fact that the minimum image size (S) used in this work is larger than $d_{S}$. PA method is not applicable to stepped surfaces but it has not bias from orientation effects [7]. Certainly, SIDS, MIDS 
L. VAZQUEZ et al.

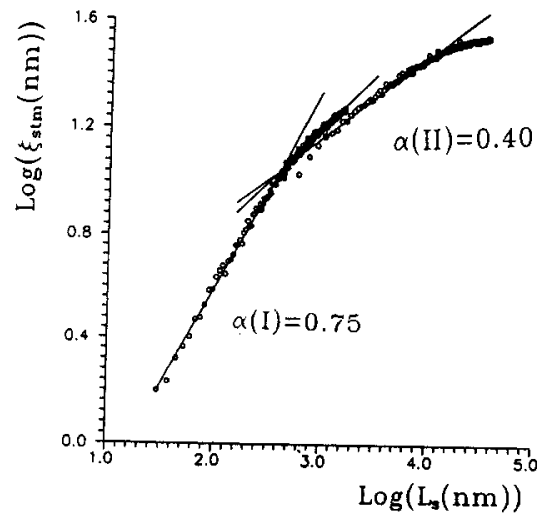

Fig3a: $\log \left(\xi_{\text {stm }}\right)$ vs $\log \left(\mathrm{L}_{s}\right)$ plot obtained by the SIDS method.

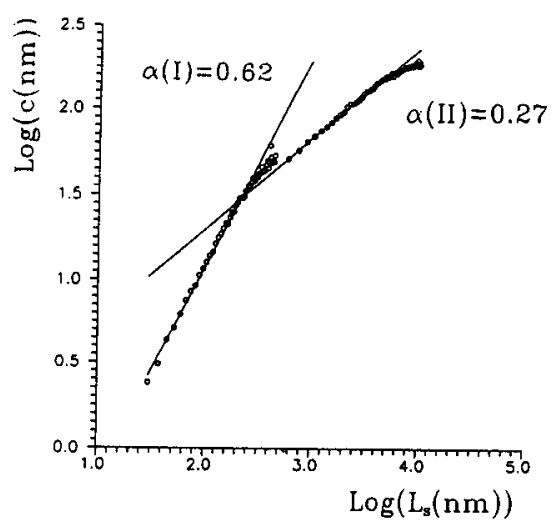

Fig3c: $\log (c)$ vs $\log \left(L_{s}\right)$ plot obtained by the $V$ method.

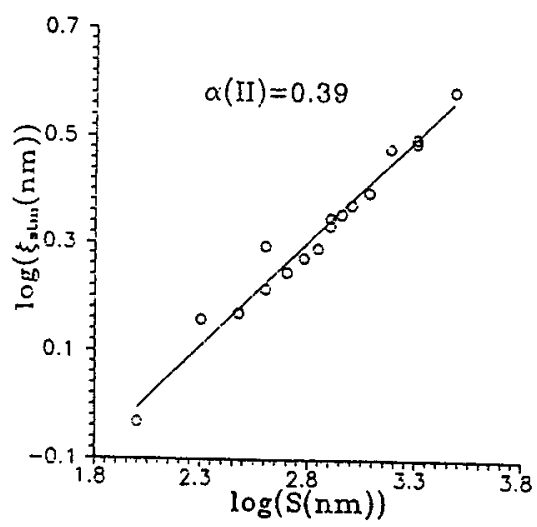

Fig3e: $\log \left(\xi_{\text {stm }}\right)$ vs $\log (S)$ plot obtained by the MIDS method.

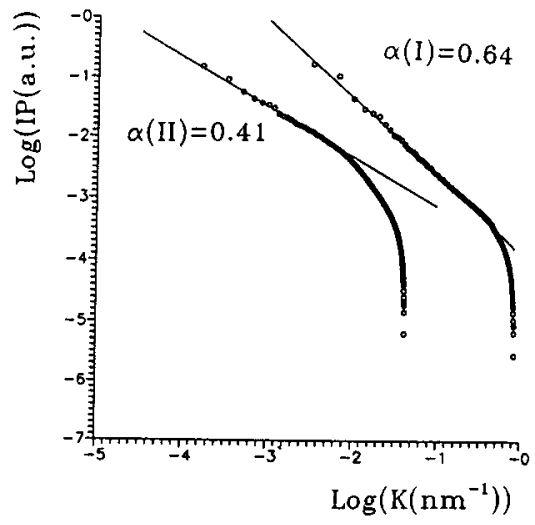

Fig3b:Log(IP) vs $\log (\mathrm{K})$ plot obtained by the FT method.

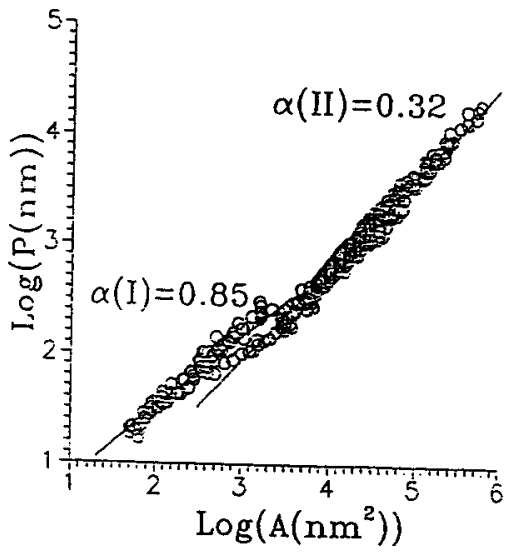

Fig 3d: $\log (P)$ vs $\log (A)$ plot ontained by the PA method.

TABLE I: Values of $\alpha(\mathrm{I}), \alpha(\mathrm{II})$ and $\mathrm{L}_{\mathrm{sc}}$ for vapor deposited Au films on glass derived from STM methods.

$\begin{array}{lrrr}\text { method } & \alpha(\mathrm{I}) & \alpha(\mathrm{II}) & \mathrm{L}_{s c}(\mathrm{~nm}) \\ \text { s-i-dynamic scaling } & 0.90 & 0.35 & 39 \\ \text { m-i-dynamic scaling } & - & 0.34 & - \\ \text { Variogram } & 0.65 & 0.28 & 20 \\ \text { Perimeter-area } & 0.75 & 0.40 & 30 \\ \text { Fourier transform } & 0.70 & 0.30 & 20\end{array}$


and $\mathrm{V}$ methods require plane substraction but they can be applied to all types of surfaces. The MIDS method appears to be a precise method as it gains more statistics than other methods employing single STM images [6]. Finally, the FT method gives the poorest results for $200 \times 200$ and $400 \times 400$ file sizes, i.e. those file sizes commonly used in STM measurements. However, this comparative study has indicated that the performance differences among the methods can be diminished by analyzing a large number of STM images. In this way similar values of $\alpha(\mathrm{I}), \alpha(\mathrm{II})$ and $\mathrm{d}_{\mathrm{s}}$ can be approached.

The value $\alpha(\mathrm{II})=0.35$ agrees with the results of large scale computer simulations of $3 \mathrm{~d}$-deposits generated by ballistic models at incident angles near normal to the substrate plane without surface diffusion [1]. This means that for $L_{S}>d_{S}$, the deposit surface behaves as a self-affine fractal. On the other hand, the value $\alpha(I)=0.8$ resulting for $\mathrm{L}_{\mathrm{s}}<\mathrm{d}_{\mathrm{S}}$, indicates that the columnar surfaces are near smooth.

In conclusion, the different methods properly applied to high quality STM images of vapor deposited gold films, lead to similar $\alpha(\mathrm{I}), \alpha(\mathrm{II})$ and $\mathrm{L}_{\mathrm{s}}$ values. Therefore, these methods provide a suitable way to characterize rough surfaces at the nanometer level. A correct physical interpretation of the results is certainly needed. For instance, the change of $\alpha$ with the image size is a clear indication of the existence of cut-offs rather than errors or limitations of the methods themselves. Besides, due to the statistical fractal characteristics of real rough surfaces and the local probe nature of the STM, a large number of samples has to be measured in order to obtain reliable $\alpha$ values.

Acknowledgement- This work was done within the frame of the cooperation program between CSIC (Spain) and CONICET Argentina

\section{REFERENCES}

1. F.Family, Dynamic Scaling and Phase Transition in Interface Growth, Physica A 168, 561-580 (1990) and references therein.

2. M.W. Mitchell and D. A. Bonnel, Quantitative Topographic Analysis of Fractal Surfaces by Scanning Tunneling Microscopy, J. Mater. Res. 5, 2244-2254 (1990).

3. J. M. Gómez-Rodríguez, L. Vázquez, A. Baró, R. C. Salvarezza, J. M. Vara and A. J. Arvia, Fractal Surfaces of Gold and Platinum Electrodeposits. Dimensionality Determination by STM, J. Phys. Chem. 96 347-350 (1992).

4. P. Herrasti, P. Ocón, L. Vázquez, R. C. Salvarezza, J. M. Vara and A.J. Arvia, STM Study on the Growth Mode of Vapor Deposited Gold Films, Phys. Rev. A 45, 7740-7747 (1992).

5. R. C. Salvarezza, L. Vázquez, P. Ocón, P. Herrasti, J.M. Vara and A.J. Arvia, Self-affine Fractal Vapor Deposited Gold Surface Characterization by STM, Europhys. Lett. 20, 727-732 (1992).

6. J. Krim, I. Hevaert, C. Haesendock and Y. Bruynseraede, STM Observation of Self-affine Fractal Roughness in Ion-bombarded Film Surfaces, Phys. Rev. Lett. 70, 57-60 (1993).

7. J.M. Williams and T.P. Beebe Jr., Analysis of Fractal Surfaces using Scanning Probe Microscopy and Multiple-image Variography, J. Phys. Chem. 97, 6249-6260 (1993).

8. B.B. Mandelbrot, The Fractal Geometry of Nature, W.H. Freeman, New York (1982).

9. R.F. Voss in Fundamental Algorithms in Computer Graphics, R.A. Eernshawed, Springer Verlag, Berlin (1985). 